\title{
Planned Clinical Study
}

National Cancer Institute

\section{Source}

National Cancer Institute. Planned Clinical Study. NCI Thesaurus. Code C70704.

A business process status of a clinical study which occurs before the study starts. A study plan on which the clinical trial is based focuses on several priority parameters that include but are not limited to the types of subjects eligible to participate in the study, targeted accrual numbers, schedules and durations of interventions, the length of the study, and the planned duration of a subject participation. These outlines are a part of a study protocol which is more a detailed and comprehensive study document. 